\title{
Tenofovir has inferior efficacy in adefovir-experienced chronic hepatitis B patients compared to nucleos(t)ide- naïve patients
}

\author{
Goh Eun Chung ${ }^{1 *}$, Eun Ju Cho ${ }^{2 *}$, Jeong-Hoon Lee ${ }^{2}$, Jeong-ju Yoo ${ }^{2,3}$, Minjong Lee ${ }^{4}$, Yuri Cho ${ }^{2,5}$, Dong Hyeon Lee ${ }^{2,6}$, \\ Hwi Young Kim², Su Jong Yu', Yoon Jun Kim², Jung-Hwan Yoon ${ }^{2}$, and Fabien Zoulim ${ }^{8}$ \\ 'Department of Internal Medicine, Healthcare Research Institute, Gangnam Healthcare Center, Seoul National University Hospital, \\ Seoul; ${ }^{2}$ Department of Internal Medicine and Liver Research Institute, Seoul National University College of Medicine, Seoul; \\ ${ }^{3}$ Department of Gastroenterology and Hepatology, Soonchunhyang University Bucheon Hospital, Bucheon; 'Department of Internal \\ Medicine, Kangwon National University Hospital, Chuncheon; 'Department of Internal Medicine, CHA Gangnam Medical Center, CHA \\ University, Seoul; ' ${ }^{6}$ epartment of Internal Medicine, Seoul Metropolitan Government Seoul National University Boramae Medical \\ Center, Seoul; ${ }^{7}$ Department of Internal Medicine, Ewha Womans University School of Medicine Liver Center, Ewha Womans University \\ Mokdong Hospital, Ewha Womans University School of Medicine, Seoul, Korea, ${ }^{8}$ INSERM Unité 1052, Cancer Research Center of Lyon, \\ Hospices Civils de Lyon, Lyon University, Lyon, France
}

Background/Aims: A recent study reported that entecavir had inferior efficacy in nucleos(t)ide analogue (NA)experienced chronic hepatitis $B(C H B)$ patients compared to NA-naïve patients. We sought to compare the efficacy of tenofovir disoproxil fumarate (TDF) in NA-experienced and NA-naïve CHB patients.

Methods: We retrospectively enrolled 252 consecutive patients who had a serum hepatitis B virus (HBV) DNA level greater than 2,000 IU/mL at the initiation of TDF treatment and who received TDF for at least 6 months. Complete virologic suppression (CVS) was defined as undetectable serum HBV DNA. We generated a multivariate Cox proportionalhazard model to examine predictive factors that were independently associated with time to CVS.

Results: The mean age of patients was 48.2 years, and the cohort included 181 NA-naïve patients and 71 NAexperienced patients. The median duration of TDF treatment was 14.4 (interquartile range, 9.5-17.8) months. A total of 167 (92.3\%) of 181 NA-naïve patients achieved CVS, and 60 (84.5\%) of 71 NA-exposed patients achieved CVS. Forty-nine (89.1\%) of 55 patients who previously took an NA aside from adefovir and 11 (68.8\%) of 16 adefovir-experienced patients achieved CVS. In multivariable analysis, previous adefovir exposure significantly influenced time to CVS (hazard ratio, 0.37; $95 \%$ confidence interval, $0.19-0.72 ; P=0.003)$, after adjusting for HBeAg positivity, baseline HBV DNA level and cirrhosis.

Conclusions: Tenofovir had inferior efficacy in adefovir-experienced CHB patients compared to NA-naïve patients. The response of patients with previous adefovir exposure to TDF monotherapy should be monitored closely.

(Clin Mol Hepatol 2017;23:66-73)

Keywords: Tenofovir; Adefovir; Hepatitis B

\begin{abstract}
Abbreviations:
anti-HBe, hepatitis B e antigen antibody; CHB, chronic hepatitis B; CVS, complete virologic suppression; $\mathrm{HBeAg}$, hepatitis $B$ e antigen; $H B V$, hepatitis $B$ virus; NA, nucleos(t)ide analogue; PCR, polymerase chain reaction; TDF, tenofovir disoproxil fumarate
\end{abstract}

\section{Corresponding author: Jeong-Hoon Lee}

Department of Internal Medicine and Liver Research Institute, Seoul National University College of Medicine, 103 Daehak-ro, Jongno-gu, Seoul 03080, Korea

Tel: +82-2-2072-2228, Fax: +82-2-762-9662

E-mail:pindra@empal.com or jhleemd@gmail.com

\footnotetext{
* The first two authors contributed equally to this study.
} 


\section{INTRODUCTION}

Chronic hepatitis B (CHB) is a global health problem because of its potentially serious outcomes, including cirrhosis, hepatic decompensation, and hepatocellular carcinoma. The primary goal of CHB therapy is to prevent disease progression and prolong survival through long-term suppression of hepatitis B virus (HBV) replication. ${ }^{1-3} \mathrm{~A}$ recent cohort study reported that entecavir failed to show comparable efficacy in nucleos(t)ide analogue (NA)-experienced CHB patients and NA-naïve patients, ${ }^{4}$ which suggests the important role of previous treatment history in patients with CHB.

Tenofovir disoproxil fumarate (TDF) has been recommended by international guidelines for the treatment of CHB with or without NA-resistant mutations. ${ }^{1,2}$ TDF has demonstrated strong, longterm antiviral efficacy in treatment-naïve CHB patients, ${ }^{5}$ and TDF therapy has also shown efficacy in CHB patients with altered responsiveness to lamivudine and adefovir. ${ }^{6}$ Additionally, TDF showed comparable efficacy in lamivudine-experienced and NAnaïve patients, irrespective of the presence of NA-resistant mutations. ${ }^{7}$ A retrospective study reported that TDF therapy has excellent long-term antiviral efficacy, even in NA-experienced patients with previous treatment failure. ${ }^{8}$

Currently, no guidelines exist regarding the selection of an antiviral agent in NA-experienced patients without detectable genotypic resistance, ${ }^{9,10}$ these patients are frequently treated as NAnaïve patients in clinical practice. Therefore, we conducted a retrospective cohort study to compare treatment responses and durations of TDF in NA-experienced CHB patients and NA-naïve patients.

\section{MATERIALS AND METHODS}

\section{Study population}

This retrospective cohort study included NA-naïve and NA-experienced CHB patients who were treated with TDF monotherapy between 2010 and 2013 at Seoul National University Hospital (Seoul, Korea). We included CHB patients with baseline serum HBV DNA levels of greater than $2,000 \mathrm{IU} / \mathrm{mL}$ who were treated with TDF for more than 6 months. Four patients who had a history of liver transplantation were excluded from the study and there was no coinfection with hepatitis $C$, hepatitis $D$, or human immunodeficiency virus. All patients were evaluated every 3 to 6 months with biochemical and virologic assessments, which in- cluded the presence of hepatitis B e antigen ( $\mathrm{HBeAg}$ ) and antibody and serum levels of alanine aminotransferase, HBV DNA, and creatinine. Genotypic mutation analysis was performed at baseline and in cases of virologic breakthrough, which was defined as an increase in HBV DNA levels of at least $1 \mathrm{log} \mathrm{IU} / \mathrm{mL}$ compared to the nadir, or incomplete response, which was defined as detectable HBV DNA after 6 months of therapy. The study was approved by the Institutional Review Board of the Seoul National University Hospital; the requirement for informed consent was waived.

\section{Study endpoints and measurements}

The primary endpoint of this study was complete virologic suppression (CVS), defined as an HBV DNA level less than $20 \mathrm{IU} / \mathrm{mL}$, as determined by quantitative polymerase chain reaction (PCR) assay. ${ }^{11,12}$ We also evaluated factors that predicted or influenced the rate of CVS.

Serum HBV DNA levels were measured using either the Abbott m2000 (Abbott Diagnostics, Chicago, IL, USA) quantitative PCR assay, which has a lower limit of detection of $15 \mathrm{IU} / \mathrm{mL}$, or the Roche COBAS TaqMan (Roche Molecular System, Branchburg, NJ, USA) quantitative PCR assay, which has a lower limit of detection of $20 \mathrm{IU} / \mathrm{mL}$. Direct PCR-based DNA sequencing was performed to identify HBV polymerase gene mutations conferring resistance to lamivudine (rtM204V/I/S, rtL180M), adefovir (rtA181T/V, rtN236T), and entecavir (rtL180M + rtM204V/I \pm rtl169T \pm rt$\mathrm{V} 173 \mathrm{~L} \pm \mathrm{rtM} 250 \mathrm{~V} / / / \mathrm{L} / \mathrm{M} \pm \mathrm{rtT} 2184 \mathrm{~S} / \mathrm{A} / / / \mathrm{L} / \mathrm{G} / \mathrm{C} / \mathrm{M} \pm \mathrm{rtS202} / / \mathrm{G} \pm$ I163V/A186T).

\section{Statistical analysis}

Continuous variable values were reported as median value with interquartile range, unless all are normally distributed. We used the Student's $t$-test or Mann-Whitney test to compare continuous variables between NA-experienced and NA-naïve patient groups, and we used the chi-square test or Fisher's exact test to compare categorical variables. We used analysis of variance to compare continuous variables. We performed multivariate Cox regression analysis to analyze the association between previous exposure to antivirals and CVS during TDF treatment, while controlling for potential confounders. Statistical analysis was performed using SPSS statistics software for Windows, version 19.0 (IBM Corp., Armonk, NY, USA). $P$-values less than 0.05 were considered statistically significant. 


\section{RESULTS}

\section{Study population}

A total of 252 patients started TDF therapy during the study period. The mean ( \pm standard deviation) age of the patients included in our study was $48.2( \pm 11.5)$ years, and $129(51.2 \%)$ of the patients were male. The median duration of TDF treatment was 14.4 months (interquartile range, 9.5-17.8) and the median follow-up duration was 14.5 months (interquartile range, 9.5-17.8). Table 1 summarizes the baseline clinical and laboratory characteristics according to treatment group. The study population included 181 NA-naive patients and 71 NA-experienced patients. Patients in both groups had similar baseline characteristics. Pretreatment HBV DNA level and HBeAg positivity rate were higher in the NAexperienced group than in the NA-naiive group, but the difference was not significant ( $P=0.40$ and $P=0.08$, repectively). The presence of cirrhosis and the duration of TDF treatment were not significantly different between the two groups ( $P=0.49$ and $P=0.58$, respectively). Among the 71 NA-experience patients, 9 patients showed suboptimal response to prior NA treatment and 17 experienced virologic breakthrough; the remaining 45 patients had no history of either incomplete response or virologic breakthrough. Further, in this group, there were 57 with no drug resistance, 8 with lamivudine resistance, 3 with entecavir resistance and 6 with multiple drug resistant mutants. Table 2 describes the presence of

Table 1. Baseline characteristics by treatment group

\begin{tabular}{|c|c|c|c|}
\hline & NA-naïve ( $n=181)$ & NA-experienced $(n=71)$ & $P$-value \\
\hline Age (years) & $48 \pm 12$ & $49 \pm 12$ & 0.51 \\
\hline Male, n (\%) & $99(54.7)$ & $45(63.4)$ & 0.21 \\
\hline Serum ALT (IU/L) & $83(48-165)$ & $91(49-183)$ & 0.76 \\
\hline Serum creatinine (mg/dL) & $0.8(0.5-2.1)$ & $0.9(0.5-1.2)$ & 0.63 \\
\hline Serum HBV DNA $\left(\log _{10} I \mathrm{I} / \mathrm{mL}\right)$ & $6.3(5.2-7.4)$ & $5.9(3.4-8.2)$ & 0.40 \\
\hline HBeAg-positive, n (\%) & $65(37.8)$ & $34(50.0)$ & 0.08 \\
\hline Presence of cirrhosis, n (\%) & $99(54.7)$ & $38(54.3)$ & 0.95 \\
\hline \multicolumn{4}{|l|}{ Prior treatment with LAM } \\
\hline LAM-experienced & & $57(80.2)$ & \\
\hline LAM resistance at baseline & & $14(19.7)$ & \\
\hline \multicolumn{4}{|l|}{ Prior treatment with ADV } \\
\hline ADV-experienced & & $13(18.3)$ & \\
\hline ADV resistance at baseline & & $3(4.2)$ & \\
\hline Duration of current treatment (months) & $14.3(8.6-17.9)$ & $14.5(10.0-17.6)$ & 0.69 \\
\hline
\end{tabular}

Mean age data are given as mean \pm standard deviation. Unless otherwise indicated, data are medians, and data in parentheses are interquartile ranges. Liver cirrhosis was diagnosed when the platelet count was below $100,000 / \mathrm{mm}^{3}$ and associated splenomegaly or esophageal-gastric varices were detected. $N A$, nucleos(t)ide analogue; ALT, alanine aminotransferase; HBV, hepatitis B virus; HBeAg, hepatitis B antigen; LAM, lamivudine; ADV, adefovir.

Table 2. Baseline polymerase sequence mutations

\begin{tabular}{|c|c|c|c|c|}
\hline Sites of resistant mutation & Drug & $\begin{array}{c}\text { NA except } \\
\text { ADV-experienced }\end{array}$ & ADV-experienced & Total \\
\hline M204l & LAM & & 1 & 1 \\
\hline A181V & LAM/ADV & & & 0 \\
\hline L180M+M204I/V & LAM & 3 & 1 & 4 \\
\hline L180M+M204V+V173L & LAM & & 3 & 3 \\
\hline L180M+M204I/V $\pm T 184 \pm S 202$ & LAM/ETV & 1 & 2 & 3 \\
\hline L180M+M204I/V+S202+A181T/V & LAM/ETV/ADV & & 2 & 2 \\
\hline L180M+M204I/V+N236T & LAM/ADV & & 1 & 1 \\
\hline
\end{tabular}

$N A$, nucleos(t)ide analogue; ADV, adefovir; LAM, lamivudine; ETV, entecavir. 

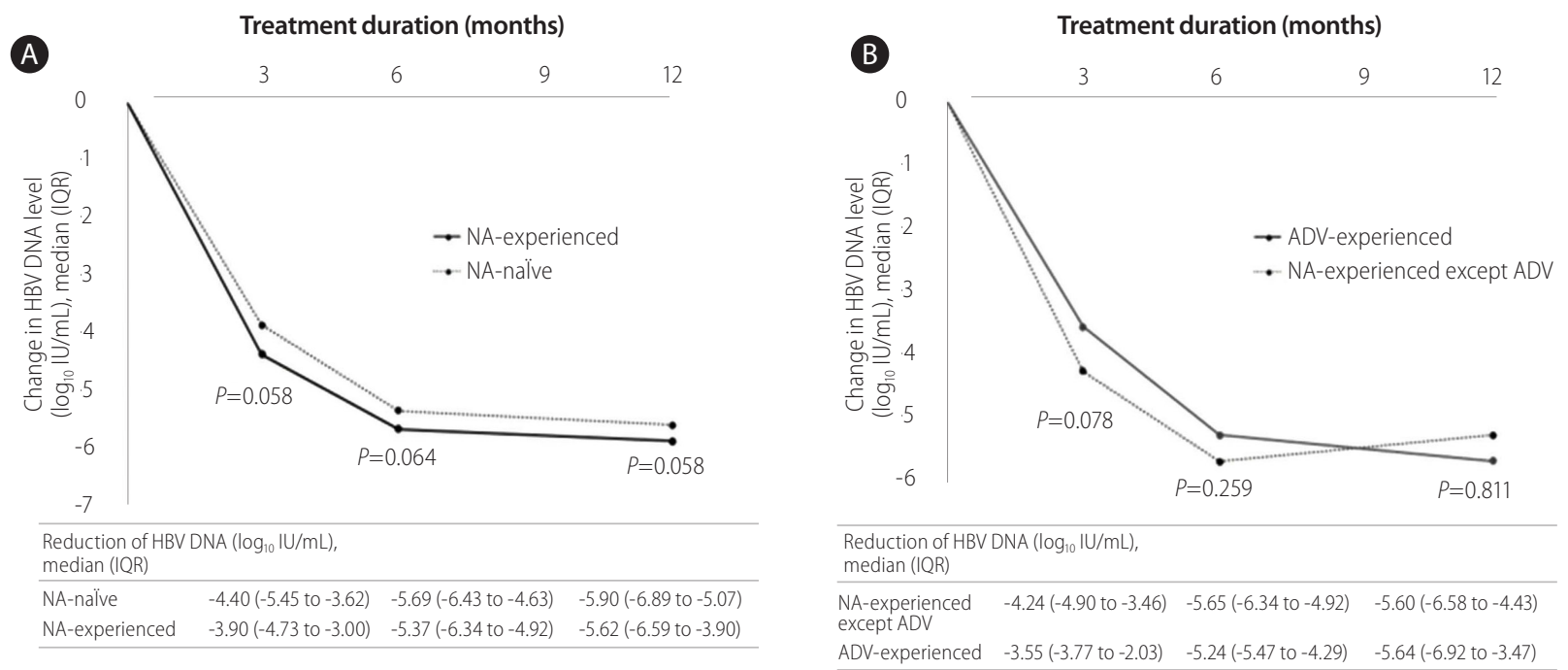

Figure 1. Treatment responses during tenofovir therapy. Changes in median log values of the serum HBV DNA levels from baseline during tenofovir therapy. An independent sample $t$ test was used for the statistical analysis at each time point. (A) NA-naïve vs. NA-experienced (B) NA-experienced except ADV vs. ADV experienced. HBV, hepatitis B virus; NA, nucleos(t)ide analogue; ADV, adefovir.

baseline polymerase gene mutations in each patient group.

\section{Treatment response to TDF and predictive factors for CVS}

Figure 1 shows the median changes in HBV DNA levels at each time point during TDF therapy. At 3 months and 12 months, the decrease in HBV DNA was marginally less prominent in the NAexperienced group than in the NA-naïve group (both $P=0.058$, Fig. 1A). However, the decrease in HBV DNA was comparable between NA-experienced except ADV group and ADV-experienced group (Fig. 1B).

A total of 167 (92.3\%) of the 181 NA-naive patients achieved CVS, and 60 (84.5\%) of 71 NA-exposed patients achieved CVS. When NA-experienced patients were further categorized into adefovir-naive patients and adefovir-experienced patients, 49 $(89.1 \%)$ of 55 with NA except adefovir-experienced patients and $11(68.8 \%)$ of 16 with adefovir-experienced patients achieved CVS. Multivariate Cox regression model revealed that previous adefovir exposure significantly influenced the time to CVS (HR, $0.37 ; 95 \% \mathrm{Cl}, 0.19-0.72 ; P=0.003)$, after adjusting for $\mathrm{HBeAg}$ positivity, baseline HBV DNA level and cirrhosis (Fig. 2, Table 3).

The TDF-induced CVS rate in adefovir-experienced patients without adefovir-resistant mutants $(10 / 13,90.9 \%)$ was higher than in adefovir-experienced patients with adefovir-resistance $(1 / 3,9.1 \%)(P=0.014$ by log rank test).

Next, we separately analyzed the impact of previous adefovir

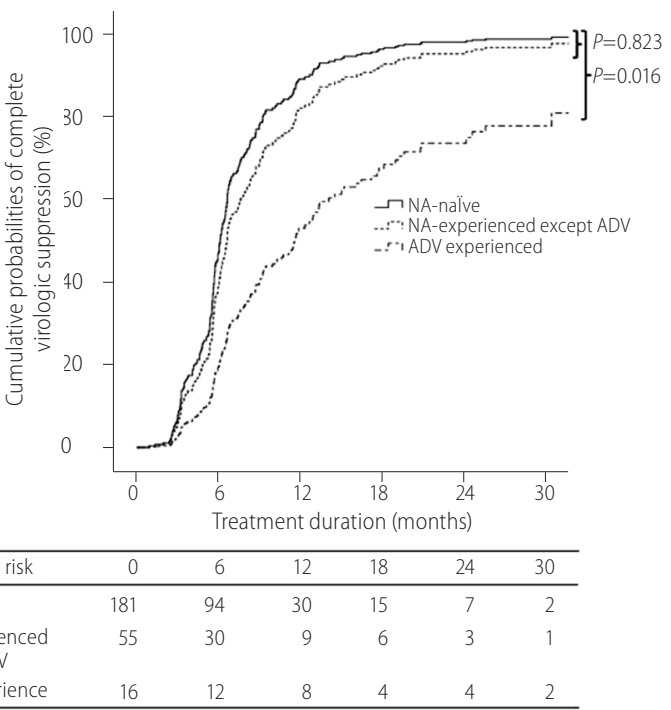

Figure 2. Efficacy with tenofovir therapy: cumulative probabilities of complete virologic suppression (undetectable serum hepatitis B virus DNA). Adefovir-experienced patients had a significantly lower likelihood of complete virologic suppression than treatment-naïve patients. NA, nucleos(t)ide analogue; ADV, adefovir.

exposure in subgroups of patients who were HBeAg-positive and HBeAg-negative. The results of the subgroup analyses revealed that the impact of previous adefovir-exposure was significant in the HBeAg-positive group ( $P=0.004$, Table 4). In the HBeAg-negative group, the CVS was lower in the adefovir-experience group, although it failed to reach statistical significance (Table 5).

During the TDF treatment period, virologic breakthrough oc- 
Table 3. Univariate and multivariate analyses of factors associated with complete virologic suppression

\begin{tabular}{|c|c|c|c|c|}
\hline \multirow{2}{*}{ Variables } & \multicolumn{2}{|c|}{ Univariate analysis } & \multicolumn{2}{|c|}{ Multivariate analysis } \\
\hline & $\mathrm{HR}(95 \% \mathrm{Cl})$ & $P$-value & $\mathrm{HR}(95 \% \mathrm{Cl})$ & $P$-value \\
\hline Age & $1.01(1.00-1.02)$ & 0.04 & - & \\
\hline Gender (male vs. female) & $0.84(0.65-1.10)$ & 0.20 & & \\
\hline Presence of cirrhosis & $1.30(1.00-1.69)$ & 0.05 & $0.90(0.69-1.19)$ & 0.48 \\
\hline HBeAg-positive & $0.33(0.25-0.44)$ & $<0.001$ & $0.44(0.32-0.60)$ & $<0.001$ \\
\hline Baseline HBV DNA $\left(\log _{10} \mid \mathrm{I} / \mathrm{mL}\right)$ & $0.75(0.69-0.82)$ & $<0.001$ & $0.75(0.68-0.83)$ & $<0.001$ \\
\hline High baseline ALT level (above ULN) & $0.92(0.64-1.30)$ & 0.62 & & \\
\hline Baseline serum creatinine (mg/dL) & $0.38(0.19-0.78)$ & 0.01 & - & 0.06 \\
\hline \multicolumn{5}{|l|}{ Previous treatment } \\
\hline NA-naïve & 1.00 (reference) & & 1.00 (reference) & \\
\hline NA except ADV-experienced & $0.96(0.70-1.32)$ & 0.82 & $0.81(0.58-1.13)$ & 0.21 \\
\hline ADV experienced & $0.43(0.23-0.80)$ & 0.007 & $0.37(0.19-0.72)$ & 0.003 \\
\hline
\end{tabular}

HR, hazard ratio; $\mathrm{Cl}$, confidence interval; HBeAg, hepatitis B antigen; ALT, alanine aminotransferase; HBV, hepatitis B virus; ULN, upper limit of normal; NA, nucleos(t)ide analogue; ADV, adefovir.

Table 4. Univariate and multivariate analyses of factors associated with complete virologic suppression in $99 \mathrm{HBeAg-positive} \mathrm{patients}$

\begin{tabular}{|c|c|c|c|c|}
\hline \multirow{2}{*}{ Variables } & \multicolumn{2}{|c|}{ Univariate analysis } & \multicolumn{2}{|c|}{ Multivariate analysis } \\
\hline & HR $(95 \% \mathrm{Cl})$ & $P$-value & $\mathrm{HR}(95 \% \mathrm{Cl})$ & $P$-value \\
\hline Age & $1.01(1.00-1.01)$ & 0.59 & & \\
\hline Gender (male vs. female) & $0.90(0.57-1.41)$ & 0.64 & & \\
\hline Presence of cirrhosis & $0.96(0.62-1.51)$ & 0.87 & & \\
\hline Baseline HBV DNA ( $\left.\log _{10} I \mathrm{U} / \mathrm{mL}\right)$ & $0.88(0.77-1.00)$ & 0.06 & $0.82(0.71-0.95)$ & 0.007 \\
\hline High baseline ALT level (above ULN) & $0.93(0.51-1.68)$ & 0.80 & & \\
\hline Baseline serum creatinine (mg/dL) & $0.33(0.09-1.25)$ & 0.10 & $0.33(0.07-1.01)$ & 0.07 \\
\hline \multicolumn{5}{|l|}{ Previous treatment } \\
\hline NA-naïve & 1.00 (reference) & & 1.00 (reference) & \\
\hline NA except ADV- experienced & $1.08(0.64-1.82)$ & 0.77 & $0.83(0.47-1.45)$ & 0.51 \\
\hline ADV experienced & $0.47(0.21-1.04)$ & 0.06 & $0.28(0.12-0.66)$ & 0.004 \\
\hline
\end{tabular}

HBeAg, hepatitis B antigen; HR, hazard ratio; Cl, confidence interval; HBV, hepatitis B virus; ALT, alanine aminotransferase; ULN, upper limit of normal; NA, nucleos(t)ide analogue; $A D V$, adefovir.

curred in two patients. Both patients had multi-drug resistance for lamivudine, adefovir, and entecavir. One patient had rtL180M, rtA181T, rtM204V, and rtS202G mutations and the other patient had rtL180M, rtA181V, rtM204V, and rtS202G mutations of HBV polymerase.

\section{DISCUSSION}

This was the first study to compare the efficacy of TDF therapy in NA-naïve and NA-experienced patients. TDF demonstrated in- ferior efficacy in adefovir-experienced CHB patients compared to NA-naïve patients. CHB patients with a history of adefovir exposure had a significantly lower CVS rate than those without previous adefovir exposure.

Patients with drug-resistant CHB have a poor prognosis compared to patients without drug resistance. ${ }^{13}$ Recently, the risk of developing NA-resistant mutations has decreased in treatmentnaïve patients after widespread use of highly potent antiviral agents with high genetic barriers, such as entecavir and TDF. ${ }^{5,8} \mathrm{~A}$ recent study showed that the efficacy of TDF was preferable to entecavir for achieving CVS in NA-experienced CHB patients with- 
Table 5. Univariate and multivariate analyses of factors associated with complete virologic suppression in 153 HBeAg-negative patients

\begin{tabular}{|c|c|c|c|c|}
\hline \multirow{2}{*}{ Variables } & \multicolumn{2}{|c|}{ Univariate analysis } & \multicolumn{2}{|c|}{ Multivariate analysis } \\
\hline & $\mathrm{HR}(95 \% \mathrm{Cl})$ & $P$-value & $\mathrm{HR}(95 \% \mathrm{Cl})$ & $P$-value \\
\hline Age & $1.01(1.00-1.03)$ & 0.36 & & \\
\hline Gender (male vs. female) & $0.83(0.90-1.16)$ & 0.28 & & \\
\hline Presence of cirrhosis & $1.00(0.70-1.42)$ & 0.98 & & \\
\hline Baseline HBV DNA ( $\left.\log _{10} \mid \mathrm{U} / \mathrm{mL}\right)$ & $0.72(0.63-0.83)$ & $<0.001$ & $0.73(0.63-0.83)$ & $<0.001$ \\
\hline High baseline ALT level (above ULN) & $0.99(0.63-1.56)$ & 0.97 & & \\
\hline Baseline serum creatinine (mg/dL) & $0.52(0.21-1.32)$ & 0.17 & $0.56(0.21-1.46)$ & 0.24 \\
\hline \multicolumn{5}{|l|}{ Previous treatment } \\
\hline NA-naïve & 1.00 (reference) & & 1.00 (reference) & \\
\hline NA except ADV- experienced & $0.94(0.62-1.41)$ & 0.76 & $0.92(0.60-1.41)$ & 0.70 \\
\hline ADV experienced & $0.83(0.31-2.23)$ & 0.72 & $0.71(0.26-1.94)$ & 0.50 \\
\hline
\end{tabular}

$\mathrm{HBeAg}$, hepatitis B antigen; HR, hazard ratio; $\mathrm{Cl}$, confidence interval; HBV, hepatitis B virus; ALT, alanine aminotransferase; ULN, upper limit of normal; $N A$, nucleos(t)ide analogue; ADV, adefovir.

out genotypic resistance, which suggests that previous antiviral treatment has clinical implications regarding the selection of an antiviral agent irrespective of preexisting genotypic resistance. ${ }^{14}$ Likewise, our previous study showed that prior treatment with low-potency lamivudine affected the long-term efficacy of entecavir, even without genotypic resistance to lamivudine. ${ }^{4}$ Drug- resistant mutations developed from previously used drugs are preserved in covalently closed circular DNA in the liver, and these mutations might attenuate the efficacy of subsequent drugs to which the virus was not previously exposed. ${ }^{15}$ We found that treatment response to TDF was significantly less pronounced in adefovir-experienced patients than in treatment-naïve patients, and this finding may have resulted from the patients with adefovir-resistance. In fact, in this study, the TDF-induced CVS rates in adefovir-experienced patients without adefovir-resistant mutants were significantly higher than those in adefovir-experienced patients with adefovir-resistance. Additionally, two patients with previous NA-exposure experienced viral breakthroughs during TDF treatment. Although TDF resistance has not yet been identified in CHB patients, our findings suggest that long-term TDF monotherapy might result in genetic resistance in adefovir-experienced patients. In fact, a case report of virological breakthrough during TDF treatment with multi-site polymerase mutations highlighted the potential risk of development of TDF-resistant mutations in patients exposed to sequential antiviral therapy. ${ }^{16}$ The treatment efficacy of highly potent antiviral agents might decrease in patients who have previous experience with low-potency NAs, so high-potency antiviral agents, such as entecavir or TDF, should be considered as first-line therapy.
There was a difference in treatment response between patients with adefovir genotypic resistance and those without adefovir resistance. In an in vitro study, the rtA181T and rtN236T double mutation reduced sensitivity to TDF by ten-fold. ${ }^{17}$ The results of another study indicated that the major adefovir-resistance mutation, rtN236T, conferred three- to four-fold reduced susceptibility to TDF in cell culture. ${ }^{18}$ In human studies, however, there is controversy regarding this issue. Patterson et al. reported that the efficacy of TDF rescue therapy following failure of both lamivudine and adefovir was inferior compared to its efficacy in treatmentnaïve patients. ${ }^{19}$ In contrast, a recent randomized controlled trial performed in Korea showed that TDF monotherapy demonstrated comparable efficacy to TDF plus entecavir combination therapy and was, therefore, a feasible treatment option for patients with adefovir-resistant disease. ${ }^{20}$ However, the follow-up duration was only 96 weeks in this study, so a longer-term follow-up study is needed to confirm these findings.

In this study, cirrhosis had a borderline association with increased CVS in the univariate analysis. However, the marginal association disappeared after adjusting for confounding factors such as HBeAg status or HBV DNA levels in the multivariate analysis. These different results may have been caused by the close negative association between $\mathrm{HBeAg}$-positivity and the presence of cirrhosis. Another explanation for the association between cirrhosis and CVS is decreased replication space in cirrhosis. Replication space for HBV has been regarded as the potential of the liver to accommodate new transcriptional templates or molecules of covalently closed circular DNA. ${ }^{21}$ In cirrhosis, decreased replication space may lead to increased viral response. 
In the current study, the efficacy of TDF was inferior in NA-experience patients compared to NA-naïve patients. In general, patients with previous exposure to low-potency antiviral agents have a slower time to CVS than NA-naïve patients, and, subsequently, NA-experienced patients have an increased risk of developing genetic resistance. Although the clinical significance of the difference in efficacy between the two groups was not substantial, our findings might support a recommendation that patients with previous exposure to low-potency antivirals should be monitored carefully and high-potency antiviral agents, such as entecavir or TDF, should be considered as a first-line therapy.

Achieving CVS is important for reducing the risks of hepatocellular carcinoma (HCC) and genotypic resistant mutation. The REVEAL-HBV study showed that the incidence of HCC increased from $0.073 \%$ per year in patients with serum HBV DNA less than $2,000 \mathrm{IU} / \mathrm{mL}$ ( $<10^{4}$ copies $/ \mathrm{mL}$ ) at entry to $0.185 \%$ per year in patients with serum HBV DNA persistence between 2,000 and $20,000 \mathrm{IU} / \mathrm{mL}\left(10^{4}-10^{5}\right.$ copies $\left./ \mathrm{mL}\right) .^{11}$ Additionally, Sinn et al. ${ }^{12}$ recently found that compensated, cirrhosis patients with low but detectable HBV DNA levels (12-2,000 lU/mL) had a higher risk of HCC than patients with undetectable HBV DNA levels $(<12 \mathrm{IU} / \mathrm{mL})$. Based on these results, CVS with HBV DNA less than $20 \mathrm{IU} / \mathrm{mL}$ might be a more appropriate endpoint than achieving HBV DNA levels below 2,000 IU/mL.

There are several limitations to our study. First, the proportion of adefovir-experienced patients was relatively small because adefovir is not usually selected as a first-line treatment due to its low genetic barrier to resistance and limited potency. The efficacy of TDF in adefovir-experienced patients was significantly lower than in treatment-naïve patients; however, since the number of adefovir-experienced patients was small, the efficacy of TDF in adefovir-experienced patients may not be estimated correctly. A larger sample size would be needed to address this limitation. Second, the presence of minor resistant HBV strains at baseline cannot be excluded. A specific strain can be detected only if it is present in more than $20 \%$ of the entire quasispecies pool in the classical clinical setting, as detected by direct sequencing. ${ }^{22}$ Thus, further study using more sensitive methods for detecting mutations (i.e., multiplex restriction fragment mass polymorphism and clonal analysis) is required. Third, more than half of the patients in our study had cirrhosis at baseline and many of the patients had been heavily treated with NA. This may indicate a referral bias, since this study was conducted in a tertiary referral center. The findings of this study might be affected by selection bias, thus, further studies are warranted to generalize our results.
In conclusion, TDF has inferior efficacy in adefovir -experienced CHB patients compared to NA-naïve patients. CHB patients with a history of adefovir exposure should be monitored closely for response to TDF monotherapy and virologic breakthrough.

\section{Funding support}

This study was supported by the Seoul National University Hospital Research Fund (Grant No. 30-2014-0070) and the Korea Health Technology R\&D Project through the Korea Health Industry Development Institute (KHIDI), funded by the Ministry of Health \& Welfare, Republic of Korea (grant number: HI16C1074).

\section{Conflicts of Interest}

The authors have no conflicts to disclose.

\section{REFERENCES}

1. Hewison M. An update on vitamin D and human immunity. Clin Endocrinol (Oxf) 2012;76:315-325.

2. Liaw YF, Kao JH, Piratvisuth T, Chan HL, Chien RN, Liu CJ, et al. Asian-pacific consensus statement on the management of chronic hepatitis B: a 2012 update. Hepatol Int 2012;6:531-561.

3. Lok AS, McMahon BJ. Chronic hepatitis B: update 2009. Hepatology 2009;50:661-662.

4. Lee JH, Cho Y, Lee DH, Lee M, Yoo JJ, Choi WM, et al. Prior exposure to lamivudine increases entecavir resistance risk in chronic hepatitis B patients without detectable lamivudine resistance. Antimicrob Agents Chemother 2014;58:1730-1737.

5. Marcellin P, Heathcote EJ, Buti M, Gane E, de Man RA, Krastev Z, et al. Tenofovir disoproxil fumarate versus adefovir dipivoxil for chronic hepatitis B. N Engl J Med 2008;359:2442-2455.

6. van Bömmel F, Zöllner B, Sarrazin C, Spengler U, Hüppe D, Möller B, et al. Tenofovir for patients with lamivudine-resistant hepatitis $B$ virus (HBV) infection and high HBV DNA level during adefovir therapy. Hepatology 2006;44:318-325.

7. Baran B, Soyer OM, Ormeci AC, Gokturk S, Evirgen S, Bozbey HU, et al. Efficacy of tenofovir in patients with Lamivudine failure is not different from that in nucleoside/nucleotide analogue-naive patients with chronic hepatitis B. Antimicrob Agents Chemother 2013;57:1790-1796

8. van Bömmel F, de Man RA, Wedemeyer H, Deterding K, Petersen J, Buggisch $P$, et al. Long-term efficacy of tenofovir monotherapy for hepatitis B virus-monoinfected patients after failure of nucleoside/ nucleotide analogues. Hepatology 2010;51:73-80.

9. European Association For The Study Of The Liver. EASL clinical practice guidelines: Management of chronic hepatitis B virus infection. J Hepatol 2012;57:167-185. 
10. Korean Association for the Study of the Liver. KASL clinical practice guidelines: management of chronic hepatitis B. Clin Mol Hepatol 2016;22:18-75.

11. Chen $C F$, Lee WC, Yang $H I$, Chang $H C$, Jen $C L$, Iloeje UH, et al. Changes in serum levels of HBV DNA and alanine aminotransferase determine risk for hepatocellular carcinoma. Gastroenterology 2011;141:1240-1248.

12. Sinn DH, Lee J, Goo J, Kim K, Gwak GY, Paik YH, et al. Hepatocellular carcinoma risk in chronic hepatitis B virus-infected compensated cirrhosis patients with low viral load. Hepatology 2015;62:694-701.

13. Liaw YF, Sung JJ, Chow WC, Farrell G, Lee CZ, Yuen H, et al. Lamivudine for patients with chronic hepatitis $B$ and advanced liver disease. N Engl J Med 2004;351:1521-1531.

14. Cho EJ, Lee JH, Cho Y, Lee YB, Yoo JJ, Lee M, et al. Comparison of the efficacy of entecavir and tenofovir in nucleos(t)ide analogue-experienced chronic hepatitis B patients. PLoS One 2015;10:e0130392.

15. Zoulim F, Locarnini $S$. Management of treatment failure in chronic hepatitis B. J Hepatol 2012:56 Suppl 1:S112-S122.

16. Lee HW, Chang HY, Yang SY, Kim HJ. Viral evolutionary changes during tenofovir treatment in a chronic hepatitis B patient with sequential nucleos(t)ide therapy. J Clin Virol 2014;60:313-316.
17. Qi X, Xiong S, Yang H, Miller M, Delaney WE 4th. In vitro susceptibility of adefovir-associated hepatitis $B$ virus polymerase mutations to other antiviral agents. Antivir Ther 2007;12:355-362.

18. Delaney WE 4th, Ray AS, Yang H, Qi X, Xiong S, Zhu Y, et al. Intracellular metabolism and in vitro activity of tenofovir against hepatitis B virus. Antimicrob Agents Chemother 2006;50:2471-2477.

19. Patterson SJ, George J, Strasser SI, Lee AU, Sievert W, Nicoll AJ, et al. Tenofovir disoproxil fumarate rescue therapy following failure of both lamivudine and adefovir dipivoxil in chronic hepatitis B. Gut 2011;60:247-254.

20. Lim YS, Yoo BC, Byun KS, Kwon SY, Kim YJ, An J, et al. Tenofovir monotherapy versus tenofovir and entecavir combination therapy in adefovir-resistant chronic hepatitis B patients with multiple drug failure: results of a randomised trial. Gut 2015;65:1042-1051.

21. Zhang YY, Summers J. Low dynamic state of viral competition in a chronic avian hepadnavirus infection. J virol 2000;74:5257-5265.

22. Lok AS, Zoulim F, Locarnini S, Bartholomeusz A, Ghany MG, Pawlotsky JM, et al. Antiviral drug-resistant HBV: standardization of nomenclature and assays and recommendations for management. Hepatology 2007;46:254-265. 